\title{
Changer ses manières de faire agir autrui à l'occasion d'expériences significatives : le cas de managers
}

\begin{abstract}
Abstrait
Les attitudes envers autrui de sujets adultes ou de personnes ayant une position forte dans la vie professionnelle sont souvent considérées comme difficiles à changer. On constate cependant que dans certaines circonstances, notamment à l'occasion d'expériences significatives dans leur propre trajectoire, des changements importants d'attitude peuvent survenir dans leur manière de gérer et faire gérer leurs interactions avec autrui. Avec un travail empirique portant sur les témoignages de ces managers sur leurs interactions avec autrui, nous illustrons les processus et les activités qui caractérisent ce changement de soi dans la relation avec les autres. Si l'apprentissage peut être considéré comme une « transformation d'habitude d'activité » (Barbier 2011 : 32), nous sommes convaincue qu'une expérience significative peut déclencher un processus de changement dans les apprentissages dédiés aux compétences humaines qui permettent aux managers de s'adapter à diverses situations multipliées par le nombre de rôles qu'ils jouent dans chaque situation.
\end{abstract}

Mots-clés : apprentissage, attitude, expérience, manager, manière.

\section{Changing One's Ways of Making Others Act after Significant Experiences: the Case of Managers}

\begin{abstract}
Attitudes towards others of adult subjects or of people with a strong position in professional life are often considered difficult to change. However, in certain circumstances, especially during significant experiences in their own trajectory,
\end{abstract}

\footnotetext{
* Conservatoire National des Arts et Métiers (le Cnam), Paris, France.

Article soumis le 2 février 2021 ; accepté pour publication le 15 février 2021.
} 
significant changes in attitude can occur in the way they manage and get to manage their interactions with others. Through empirical work on the testimonies of these managers regarding their interactions with others, we illustrate the processes and activities that characterize changes of self in relation with others. If learning can be considered as a "transformation of activity habits" (Barbier 2011: 32), we are convinced that a significant experience can trigger a process of change in learnings dedicated to human competencies that allows managers to adapt themselves to various situations multiplied by the number of roles they play in each situation.

Keywords: learning, attitude, experience, manager, manner.

\section{Introduction}

En quoi le travail du manager, est-il spécifique ? La littérature classique sur le management le caractérise par des tâches ${ }^{1}$ telles que la planification, l'organisation, la coordination et le contrôle etc. Or, au-delà du manifeste, les tâches relevant de la fonction humaine du management sont peu visibles ${ }^{2}$.

\section{Ce que disent les managers sur leurs manières de faire agir autrui}

Nous avons eu l'occasion de réaliser une enquête qualitative auprès de 12 managers d'entreprise sur leur façon de diriger leurs collaborateurs. Parmi eux nous avons retenu ceux qui ont explicité un changement net de leurs manières d'agir suite à une expérience significative.

Nous avons décidé d'utiliser une grille d'analyse unique pour distinguer 4 thèmes suivants dans chacun des cinq cas, un par un :

- Discours sur la manière ancienne/initiale ;

- Discours sur l'évènement d'influence/expérience significative ;

- Discours sur la manière reconstruite/nouvelle ;

- Discours sur la leçon tirée de l'expérience significative.

\footnotetext{
1 « Nous pouvons isoler ce qu'une personne fait parce qu'elle est un manager... Il y a cinq opérations de base dans le travail d'un manager (fixer des objectifs, organiser, motiver et communiquer, mesurer, développer les gens) » (Drucker $2012: 20$ ).

2 Meryem Le Saget énumère dans son ouvrage sur les compétences « intuitives » des managers les activités suivantes : « ... sentir les choses au-delà des faits, (...) mieux percevoir les événements, comprendre les personnes et les phénomènes de groupe, (...) se couler avec confiance dans des dynamiques émergentes » (Saget $2013:$ 9).
} 


\section{Premier cas : pouvoir et bienveillance}

\section{Discours sur la manière ancienne/initiale}

Dès le début de l'entretien le manager utilise le passé composé 'avoir eu' pour affirmer l'existence d'une manière d'agir ancienne - 'le management transversal'. Il souligne aussi le caractère habituel, répétitif de cette manière en utilisant les mots 'toujours' et 'souvent'.

Parce que moi j'ai toujours eu du management transversal. C'est-à-dire sans rapports hiérarchiques.

Il essaie, non seulement d'expliquer sa compréhension par la conjonction " c'est-à-dire », mais aussi de comparer deux manières de faire agir autrui par l'expression « ne sont pas...mais... ». Il a inséré dans son discours la manière dont il voyait le « pouvoir ». L'utilisation de l'imparfait dans la phrase «j'étais encore dans le pouvoir » souligne que c'est une manière habituelle et répétitive de voir le monde de l'individu dans le passé.

moi j'étais un peu jeune. J'étais peut-être dans le pouvoir encore, parce que je me représentais un peu une forme de client.

Lorsqu'il annonçait « j'étais jeune et j'étais dans le pouvoir encore », il décrivait sa compréhension du management : que les «ordres » devaient représenter uniquement le « pouvoir » et dans aucun cas la « bienveillance».

\section{Discours sur l'expérience significative}

Le premier manager a pu retrouver facilement l'expérience significative et surtout le sentiment qu'il a éprouvé bien que cela se soit passé il y a plus d'une dizaine d'années. Il a commencé son discours avec des phrases décrivant la lourdeur de l'environnement économique :

Très gros chantier. La société était en... difficulté en 2008, crise financière, on faisait du chômage partiel et licenciements économiques. (...) J'avais une équipe sur site... qui était... qui était soumise à rude épreuve parce que le back office était relativement... le chômage partiel... était relativement indisponible.

En écoutant les détails précis sur l'année, le chiffre des effectifs et la valeur financière d'un contrat etc. nous nous attendions à ce qu'il relate son acte de commandement ou une décision stratégique prise, en se basant sur ses 'compétences analytiques ou techniques' du management. Hors en réalité notre manager s'est mis à nous parler d'un accident tragique qui est arrivé au frère de son superviseur de chantier. 
je me suis retrouvé un samedi avant 2 semaines d'interruption complète, black-out au niveau du... de la maison mère, et j'ai eu mon superviseur, la personne qui dirige le chantier...

Il indique même précisément le jour, un samedi soir, et détaille le moyen de communication utilisé par le superviseur : un coup de fil. Il décrit notamment la caractéristique physique du superviseur, costaud, « armoire à glace », en contraste avec la manière dont ce superviseur lui a parlé au téléphone : " en larmes ». Pendant l'entretien, l'interviewé se réfère même à son divorce comme si cette évocation contribuait à avoir un effet non-négligeable sur sa sensibilité aux pertes de liens entre deux personnes proches. Nous étions étonnée de l'entendre prononcer le mot « divorce » qui semble éloigné du contexte professionnel. Puis compte tenu de l'aspect de «danger » qu'un accident grave ou un divorce peut comporter, nous avons compris que leur point commun était qu'ils faisaient partie des évènements dangereux pour les relations interpersonnelles.

\section{Discours sur la manière nouvelle/reconstruite}

Le rythme du discours du sujet augmente rapidement avec une série de verbes directifs reproduisant des « ordres » sans aucune utilisation d'adjectif.

Donc... là j'ai été assez directif. Je dis 'ok, là tu restes à l'hôtel, tu me rappelles... Moi, pendant ce temps je réserve mon billet d'avion, et je te dis quand est-ce que j'arrive'. Donc j'ai pris mon billet d'avion tout seul avec ma petite carte bleue.

L'utilisation des actes successifs du langage de commandement dans cette réplique favorise aussi l'interpellation du manager sur sa manière d'agir avant et après l'évènement. Avant l'évènement : pour un manager " transversal ", l'obligation de « donner des ordres » a été transférée vers les personnels administratifs ou vers les services dédiés au management, c'est-à-dire les "bureaux ». Alors lorsque l'évènement a eu lieu, le manager n'avait accès ni à ses supérieurs, ni aux manuels indiquant les procédures à suivre.

La décision brutale du manager, partir remplacer un superviseur expérimenté, pour diriger une centaine de personnes sur un chantier pendant trois semaines, malgré le manque d'expérience, la façon d'agir habituelle, l'inquiétude et l'angoisse. Cette réplique se conclue par la phrase :

Après je suis resté sur site pendant 3 semaines et j'ai dirigé un chantier de 100 personnes. Je n'avais jamais fait vraiment comme ça.

Le manager en développant son discours a pu non seulement reconnaitre son changement, mais aussi nous permettre de distinguer les différentes phases de sa prise de conscience et les sources d'influence sur son attitude. 


\section{Discours sur la leçon tirée}

La première 'leçon' tirée de l'expérience significative de ce manager se trouve dans la conclusion à propos de l'effet que la 'bienveillance' a eu sur sa façon de faire agir les autres :

(...) du coup toute cette bienveillance m'a fait voir différemment justement la façon de donner des ordres, la façon de les suivre. Elle m'a beaucoup influencé moi, là-dessus. Parce qu'il y avait un côté très humain.

Nous avons constaté que non seulement il catégorisait une manière d'agir ou un évènement isolé, mais il essayait aussi d'analyser sa catégorisation. Autrement dit, il essayait d'exprimer sa nouvelle manière de voir les choses dans le monde : «... il y avait du côté humain ». Dans les autres répliques, son discours est construit de manière plus expressive. Le sujet utilise des métaphores telles que : « le travail, c'était un peu une valeur refuge » ou « tous mes projets étaient mes bébés » ainsi que des concepts abstraits comme la « confiance », « complice » ou « symbiose » pour manifester son état mental à propos de choses dont il présuppose l'existence dans le monde.

\section{Deuxième cas : un conflit entre réaction naturelle et nouvelle réaction}

\section{Discours sur la manière ancienne/initiale}

Quant à l'entretien avec le second manager, la manière ancienne correspond à la manière naturelle. Après avoir écouté notre question de démarrage il a réagi spontanément par un rire. Effectivement, les mots utilisés suivant le rire constituent une base pour analyser les associations des expressions verbales et non-verbales. Le manager ensuite a exprimé son vif intérêt pour les possibilités de 'faire mieux', d'être 'les meilleurs' ou de 'maintenir la position de numéro un de l'entreprise'. Le recours soutenu au thème de l'évaluation est révélateur : sa manière initiale consistait à se concentrer sur la performance technique des activités réalisées par le manager. Il a donné un nom à sa manière de faire agir autrui : «j'ai une approche encore d'entreprise ». Ainsi, il a essayé de la décrire par une 'équation' : « il y a un problème et hop, une solution ». Cette forme d'expression verbale révèle sa manière d'agir initiale comme un réflexe spontané. Le mot 'hop' illustre très bien la spontanéité dans sa manière d'agir, qui fait écho à l'utilisation du mot « nature » dans la première réplique.

\section{Discours sur l'expérience significative}

Le manager, après avoir écouté notre demande et fait une pause de cinq (5) secondes, s'est mis à parler par une interjection marquant sa réussite de faire revenir un souvenir « $\mathrm{Ah} »$. 
Ah, voilà. Il y a eu un concert que j'ai produit il y a deux jours. (...) Oui c'est un truc plus récent, marquant... voilà... Là, j'ai du mal. Et c'est mal pris (rire).

Le premier mot « voilà » dans «Ah, voilà » a envoyé notre attention vers le mot 'concert' qui a suivi, comme s'il agissait de l'évènement marquant dont il voulait nous parler. Pourtant, quand il a dit une deuxième fois le mot "voilà », nous avons senti que c'était pour se rassurer lui-même : "Oui, c'est un truc plus récent, marquant... voilà ». L'association des mots : « mal, mal pris » au thème « truc marquant » nous a montré le lien entre l'état affectif causé par l'expérience significative et la mémoire sélective du manager. Jusque-là, le manager n'avait parlé que de lui, de son acte et de sa nature. Sans pouvoir nommer concrètement l'évènement, il utilisait le mot «truc » et puis il nous a expliqué :

Et parce que j'ai reçu un sms immédiat de la pianiste, qui m’a dit : ton e-mail m’a beaucoup blessée.

L'occurrence soudaine du « message de la pianiste » introduisait un tiers. Le message de la pianiste avec la métaphore «blessée » a réussi à nous faire entrer dans le champ de réflexion de ce manager.

\section{Discours sur la manière nouvelle/reconstruite}

Dans ce deuxième cas, il est très intéressant pour nous de voir que les expressions verbales illustrent un conflit, une dissonance cognitive entre la 'nature' et la manière d'agir reconstruite de ce manager. Nous avons vu ici qu'il ne souhaitait pas changer sa manière naturelle, mais il l'a pourtant fait. Ce processus a commencé dès le moment où le manager a reçu le message de la pianiste.

Oui, je l'ai appelée et j'ai dit : Écoute euh... (je fais profil bas), c'est ma faute... Je n'aurai pas dû de t'envoyer ça, ce n'était pas ça ce que je voulais dire... (rire). Mais là on est sur de l'ultra sensibilité avec une personne. En plus... Elle sort à peine depuis deux ans d'une période de sa vie, très difficile...

L'alternance entre les paroles qui nous ont été adressées et celles qui s'adressait à lui-même nous a révélé que le sujet s'était obligé de changer sa manière 'naturelle'. Le discours sur la vie de la pianiste, sur son talent et ses difficultés occupe une bonne partie de la réplique suivante. Cela explique le processus par lequel le sujet trouve le moyen de diminuer sa dissonance.

Je la connais à peine, mais c'est une personne qui s'ouvre très vite sur toute sa vie et notamment sur la dernière difficulté qui s'est passé dans sa vie privée, pas dans sa vie professionnelle. Donc on est sur une très grande pianiste, qui a disparu de la circulation pendant cinq ans. Donc elle revient mais il y a un mélange d'ego, 
d'émotion naturelle plus un passif où elle a besoin de prendre une revanche ... etc.

Donc je fais marche arrière. Je dis désolé...

Le processus de la reconstruction de la manière d'agir de ce manager ne se passe pas aussi facilement. Il continue d'opposer l'approche d'entreprise à l'approche d'artiste. La dissonance cognitive entre son mode de faire agir par « ordre » et la nouvelle manière par céder autrui dont il s'est forcé à adopter persiste.

Oui, très difficile, bien sûr (rire). J'ai mon ego...

La locution « du coup » nous a montré l'arrivée d'un autre changement dans la façon d'agir du sujet. Cette fois, plutôt qu'agir spontanément en suivant sa manière 'naturelle', il a décidé d'échanger avec une deuxième personne, sa femme :

Et du coup, j'ai échangé avec elle d'abord. (...) Euh... donc c'est dur...

\section{Discours sur la leçon tirée}

L'une des particularités de ce manager est que ses réflexions se sont télescopées et mêlées les unes aux autres. Le sujet a évoqué sa manière ancienne d'agir en même temps que l'évènement marquant. Il a cité également sa réflexion autour de l'expérience significative tout de suite après avoir fait allusion au message de la pianiste.

Ça n'est pas très bien vu dans l'art. En musique, on n'aime pas des ordres. Le moment quand ils jouent, ils jouent ce qu'ils sentent. Quand ils jouent, ils jouent ce qu'ils sont. Je pense ah. C'est à peu près ça. Et donc, si tu critiques ce qu'ils jouent, tu critiques ce qu'ils sont.

Il est intéressant de noter que le sujet n'utilise pas le pronom de la première personne. Il utilise la plupart du temps les pronoms de la troisième personne : ça, on, ils. Et quand il parle de lui, il utilise le pronom de la deuxième personne « tu ». Ainsi, nous avons vu dans son discours sa leçon tirée ${ }^{3}$ à travers les actes, les sentiments des artistes, de sa femme, bref d'autrui plutôt que du sujet lui-même. La façon humoristique qu'il utilise est aussi très particulière.

Parce que je me suis fait remonter d'abord par ma femme, qui était en copie de l'email. Elle a dit : tu as fait une grosse connerie...

\footnotetext{
3 « Le discours le plus subjectif peut se parer d’une apparence d’objectivité ; l'énoncé est alors présenté comme une démonstration universellement pertinente et non comme un argumentaire assumé par un sujet » (Seignour $2011: 34$ )
} 
Nous avons observé pourtant un processus de reconstruction fait de ruptures, de conflits et surtout de combats intérieurs entre la manière naturelle et la manière nouvelle. Ce n'est pas le cas d'un processus d'apprentissage d'une compétence technique.

\section{Troisième cas : difficultés techniques/difficultés humaines}

\section{Discours sur la manière ancienne/initiale}

Concernant le troisième manager, nous n'avons trouvé la façon dont il avait fait usage au début de sa carrière que vers la fin de l'entretien :

J'ai commencé très jeune. Et très jeune j'ai encadré des personnes, mais je ne savais pas à l'époque qu'il y avait un management opérationnel. Il faut entraîner l'autre mais sans autorité.

Lorsque l'autorité fait défaut, il est possible de recourir à la méthode de la 'persuasion' (" convaincre de faire »). Ici, nous avons constaté que sa manière initiale reposait sur l'opinion de la 'masse'. Si l'on compare sa manière initiale de faire agir les autres avec la 'méthode' de son ancien directeur technique, il apparait évident que notre sujet n'avait jamais pensé exercer un commandement pour faire agir autrui :

Non ça n’a pas été mon cas... Mon directeur technique, pour lui, c'était très facile en réunion ou à l'atelier de commander les gens. De par sa stature dans l'entreprise, il dit et les gens font, il n'a pas besoin de négocier.

Il démontre, du point de vue de ce jeune cadre, l'importance croissante de l'interaction entre un manager et autrui.

\section{Discours sur l'expérience significative}

Le troisième manager nous a conduit sans détour à l'évènement qu'il considère comme l'expérience « la plus critique » dans sa carrière. En dépit du temps écoulé, ce manager réussit à l'en extraire et à décrire avec de multiples détails :

Donc je dirais que je gérais il y a quelques années, un projet technique sur le site, (...) Il y avait un flexible qui s'est abimé avec un fluide à très haute température donc avec un danger pour l'homme. (...) A l'époque j'avais pris la décision d'arrêter la production. ... Donc j'étais tout seul à maintenir cet avis-là. Donc c'était une situation qui a été assez dure à tenir.

Les mots utilisés sont révélateurs du 'combat intérieur' qui se livre dans les pensées de ce manager. La mixité des usages des thèmes techniques et des thèmes 
humains dans son discours nous a montré que dans ses réflexions, ce manager alternait entre la difficulté technique et la complexité managériale. Le manager a pu se souvenir de détails concrets tels que la cause de l'accident, mais également et surtout du nombre des personnes concernées, des questions que les autres membres lui ont posées ainsi que des doutes et du niveau de pression ressenti autour de lui. La mémoire sélective de ce manager semble privilégier la situation qui lui a permis de libérer le plus de sentiments en lui comme les sentiments de pression :

Pour moi, tout de suite, ... c'est la [situation-la] plus critique qui vient, pour moi, parce qu'arrêter un site où on était avec à peu près 80 personnes à l'époque et le directeur du site qui n'était pas là... Donc voilà, il y avait une pression qui a été assez importante.

La pression significative exercée sur ce manager dans une telle situation de crise pourrait s'expliquer par le fait que tous les points sur lesquels il pouvait s'appuyer se sont retournés contre sa prise de décision : l'absence du directeur du site, le refus de la prise de décision du directeur adjoint, les doutes des autres membres de la direction et enfin les conséquences économiques critiques engendrées par la décision qu'il voulait prendre. Lorsque tous ces éléments sont réunis, ils peuvent générer non seulement un cumul de pressions, mais aussi provoquer une rupture dans la relation entre le sujet et les autres membres de la direction. Cette rupture donne lieu à de fortes réactions physiques, intellectuelles ou bien émotionnelles.

\section{Discours sur la manière nouvelle/reconstruite}

Dans une séquence de discours de ce manager, nous avons trouvé quelques expressions intéressantes :

En fait, c'était difficile parce que derrière... j’ai parlé à des personnes qui avaient le même niveau hiérarchique que moi dans la société. L'idéal c'est de les convaincre de suivre ma décision, mais face à l'urgence, je ne peux pas convaincre tout le monde. Quand on est en face à une urgence, je dois faire comme moi je le sens, mais avec tous les risques qui peuvent être derrière. Car si je me trompe et si je n'entraine personne à suivre ma décision, je me trouve tout seul. Et bon, se tromper tout seul c'est différent de se tromper à plusieurs.

Le mot « derrière » répété dans deux phrases, renvoie aux mots « face » cités plus loin. Les couplages du mot « idéal » = « convaincre » fait implicitement écho au mot « non-idéal » non verbalisé dans la négation «ne peux pas convaincre ». Les associations de mots sont révélatrices de deux approches souvent distinctes et opposées dans le cadre des relations multidimensionnelles : spatiale, temporelle, hiérarchique, qualitative et quantitative. 
A travers un exemple récent et concret, ce manager nous a décrit une situation au travail qui illustre la dissonance $\operatorname{cognitive}^{4}$, le conflit caché entre son avis individuel et l'avis du groupe :

Oui, récemment c'est arrivé, on a interdit les téléphones portables sur le site de production... Donc du coup moi j'ai dû communiquer dessus, mais je ne suis absolument pas d'accord avec ça... Donc du coup je suis complétement contre en fait. Je suis complétement contre, mais voilà j'ai dû communiquer dessus... Alors que je ne le partage pas du tout.

Dans cette réplique, nous avons pu recueillir trois façons d'exprimer sa contestation à l'égard de la décision du groupe : " absolument pas d'accord », " complétement contre », " ne partage pas du tout ». En rapprochant les verbes utilisés, il apparait que ces liens sont tissés avec une logique évolutive. Sa réplique, plus loin, éclaire cette interrogation :

En fait, c'est avec lui [son supérieur] et les autres membres de la direction que j'en débats. Quand on fait des réunions, avec moi-même ou encore avec les autres membres cadres dans la société, on en débat etc. ou on en sorte de gérer les choses, mais on en débat qu'entre nous...

Le verbe « débattre » résume parfaitement la manière reconstruite de ce manager. La différence de sa nouvelle manière réside dans le caractère de l'interaction entre le sujet et un 'adversaire'.

\section{Discours sur la leçon tirée}

Dans ce troisième entretien le manager évoque comme 'leçon' l'importance que ses collaborateurs accordent à la compréhension du sens de leur travail et, dans le même temps, l'importance que lui-même accorde à l'opinion des tiers. Si sa manière initiale était de convaincre les autres et de chercher l'accord des autres, dans cette partie du discours, il accepte que les autres ne soient pas d'accord avec lui.

Pour moi, le plus important quand je donne une mission à une personne de mon équipe : d'abord je m'assure qu'il a bien compris, qu'il a bien compris le contexte, pourquoi qu'il faut le faire. Pour moi, ça me semble important. Ils ont beau ne pas être d'accord. Ils peuvent ne pas être d'accord etc.

Grâce à son discours, ce manager nous a montré le processus d'accumulation du savoir. L'apport d'opinions et d'arguments différents de ses collègues lui permet de nourrir ses nouvelles connaissances.

\footnotetext{
${ }^{4}$ Selon la théorie de la dissonance cognitive développée par Leon Festinger (1957), la manipulation comportementale peut amener l'individu à modifier son attitude en le 'forçant' à se comporter de manière opposée à son attitude initiale, puis rationaliser son comportement en remplaçant l'attitude initiale par une nouvelle attitude.
} 
Je pense que c'est efficace. Parce que c'est euh... Je pense que c'est mieux d'avoir

l'approbation de la personne que de lui imposer.

Les réitérations de « je pense » dans cette réplique témoignent qu'il s'agit d'un discours révélateur du nouveau regard qu'il porte sur le monde. L'opposition entre " avoir l'approbation » et "imposer » indique que la réflexion constitue effectivement une autre forme combinée d'accord et de compréhension.

Au final j'arrive toujours à entraîner les gens ou à trouver une situation, un compromis qui fait qu'on atteint l'objectif... Donc pour revenir à la première situation, ce n'était pas évident.

A la dernière réplique, le retour soudain du manager à l'expérience significative avec la conclusion 'ce n'était pas évident' nous montre que la reconstruction d'une façon qui est déjà très établie peut ne pas être facile.

\section{Quatrième cas : management direct/management indirect}

\section{Discours sur la manière ancienne/initiale}

Ce thème a commencé uniquement après une dizaine de répliques très génériques :

J'étais jeune ah. Je ne comprenais pas quelle était la difficulté d'arriver dans une concession et de leur dire qu'ils allaient faire ça, qu'ils signent, voyez. Mais pour le directeur : 'Wow ! T'as réussi qu'ils signent ce document avec ce groupe-là ! C'est bien!'

Notre attention a été attirée par des oppositions d'attitude de deux acteurs : le sujet qui n'était pas encore un manager et son directeur. Une seule et même action a été décrite de deux façons différentes : pour notre sujet (non-manager) l'accent était mis sur un objet (le document) ; pour le directeur l'accent était mis sur la relation entre « ils » et « avec ce groupe-là ». Le niveau évoluant de compréhension du travail d'un manager est un point intéressant. Les mots 'je ne comprenais pas' répétés deux fois et son explication : «j'étais jeune ah » soulignaient qu'il faisait agir autrui sans être informé de la nature de la 'difficulté' rencontrée en tant que directeur.

\section{Discours sur l'expérience significative}

Le manager nous a donné son expérience significative avec la locution de temps "Et un jour », suivie d'un énoncé décrivant le déroulement rapide de l'évènement représenté par le pronom «ça ». Ce faisant, il a amplifié l'apparition d'un événement significatif et en fait le thème dominant de cette séquence : 
Et un jour, ça s'est passé rapidement. Il y a eu une lettre qui est allée au réseau [la concession] avec la nouvelle distribution de zone géographique de chaque concession. ... Je l'ai lu. J'ai dit : 'Ah, ça a été changé'.

Nous décelons que ses premières réactions étaient formulées sans aucun signe d'affect. Phrases simples et courtes, ne contenant aucun adverbe émotionnel, énumérant uniquement les actions perceptives : recevoir, lire. Pourtant, à partir du moment où le sujet a commencé à se livrer (" se dire »), l'interjection 'Ah' et l'affirmation "ça a été changé » ont bien marqué l'apparition des affects dans ses répliques suivantes :

Et quand je suis arrivé dans cette concession, le manager [de la concession] m'avait demandé de me voir immédiatement. Il était furieux que j'aie changé, moi, [la marque a], changé sa zone géographique. Pourquoi ? (...) Alors il était furieux... Il était complétement en colère et tout.

Ce n'est qu'à son arrivée dans la concession, après avoir constaté l'état émotionnel du directeur de la concession, que sa propre réaction a été stimulée. Dans une telle situation tendue, si notre sujet était capable non seulement de percevoir les informations sur les actions de la marque, mais aussi de comprendre le sentiment de l'autre et surtout de celui qui était en train de "l'accuser ", c'était effectivement le signe qu'il avait finalement franchi une étape vers l'acquisition d'une nouvelle manière d'agir. Si les verbes caractérisant la manière initiale d'agir de ce manager sont les verbes nécessitant un seul acteur (lire, se dire...), nous avons constaté ici l'apparition d'un deuxième groupe des verbes qui nécessite d'un autre acteur, tels que : expliquer, aider, montrer, comprendre. Avec cette réplique, nous discernons le premier pas de notre sujet vers une interaction exigeant la participation de nombreux autres acteurs.

\section{Discours sur la manière nouvelle/reconstruite}

Dans ce cas, l'interlocuteur s'est beaucoup concentré sur sa perception du « management indirect » par rapport au « management direct » qu'il a associé au mot « ordres».

Alors, dans mon parcours, j'ai managé indirectement plusieurs personnes... Alors je n'avais pas une équipe directe, mais j'avais une équipe indirecte qui était composé de 10 à 15 concessions entre managers commerciaux, directeurs commerciaux et titulaires. Chacun à son niveau. D'accord ? (...) Alors c'était un travail surtout d'influence.

Le manager énonce une définition intéressante du management indirect : « un travail d'influence », ce qui sous-entend des actions exercées sur les acteurs et leurs 
actions. Nous pensons que cette logique explique pourquoi les actions de ce manager cessent d'être répétitives ou procédurales.

s'ils faisaient une chose qui n'était pas correcte, c'est moi qui allais la corriger ou c'est moi qui allais la valider ou pas. Mais peut-être ils faisaient ce que leur directeur leur avait demandé. Parce que ce n'est pas un management direct.

Après la séquence axée sur le niveau de sa compréhension du management indirect, notre sujet s'est orienté vers l'illustration de sa pratique. Il a présenté une deuxième situation au cours de laquelle se manifestent de plus nombreux acteurs : le sujet, les directeurs de la marque, les directeurs de la concession (père et fils), le personnel, les clients et les fournisseurs, etc.

J'étais manager régional du R. Il fallait qu'elle participe à des évènements commerciaux (...) Ils ne participaient jamais, parce que c'était trop cher. J'avais réussi à les convaincre de participer (...) J'ai participé à tous la mise en œuvre de la page publicitaire dans la revue presse et aussi dans la publicité pour la télé locale. Alors il fallait que je 'lead' avec le fils et le père. Et ça c'était la plus grande difficulté.

Ceci représente un long 'monologue' riche en actions diverses, incluant les détails et les résultats, les relations interpersonnelles et les dimensions multirelationnelles de son travail. En explorant la technique d'analyse thématique, on observe que l'utilisation des verbes avait également changé. Si les verbes utilisés dans les premières répliques privilégient un seul acteur, par la suite le discours du sujet propose une autre formulation dominée par un verbe : " participer ». C'est un verbe qui montre que la manière nouvelle de ce manager est une manière multirelationnelle et interpersonnelle.

\section{Discours sur la leçon tirée}

Le quatrième manager apporte une réponse positive en ce qui concerne le fait de donner des ordres comme leçon.

(pause de 5 secondes). J'aime donner des ordres, mais de manière que ce soit bien perçu par la personne, d'accord. Ce n'est pas un ordre autoritaire, mais (...) j'aime diriger un travail ou un processus. Mais alors ce n'est pas un ordre de type : «faire ci (...) comme ça ». Voilà. Vous comprenez ? (...) Le mot ordre est très général. Et on peut l'interpréter de diverses manières.

Dans cette première réplique, sont également cités plusieurs 'mais' et 'd'accord ?' ou 'vous comprenez ?'. Le connecteur 'mais' et les compléments de précision ou de vérification qui sont très présents dans chaque phrase, font penser que l'attitude favorable aux 'ordres' de ce manager ne représente pas un caractère (« autoritaire »), 
ni un acte de langage (" faire ci... comme ça »), mais résulte d'un processus complexe, qui n'est pas simple à comprendre ("Vous comprenez ? ») ou même à interpréter (« on peut l'interpréter de diverses manières »). Ensuite, selon lui, la manière de faire agir autrui en donnant des ordres comportent aussi divers niveaux.

Mais par contre, l'ordre à divers niveaux. Ça veut dire que pour un manager il commence à expliquer le travail, demander à son équipe et il directionne. Mais après, si par exemple, si ça ne passe pas, là il y a une... il faut un ordre.

Les verbes ont été classés en deux catégories, ce qui nous a permis de comprendre à quels niveaux il faisait référence :

- Niveau 1 (absence de réaction d'autrui) : commencer, expliquer, demander, directionner (diriger) il demande et il 'directionne' (dirige) ;

- Niveau 2 : («si ça ne passe pas ») : il faut un ordre.

\section{Cinquième cas : management à distance/management en présence}

\section{Discours sur la manière ancienne/initiale}

Avec le cinquième manager, nous avons noté deux styles différents : a) lorsqu'il parle de lui-même, l'affirmation de la nature de l'action solitaire se traduit par l'utilisation répétitive du pronom personnel 'je' au début de l'entretien : 'J'ai créé un magazine', 'J'ai créé une équipe' ; b) lorsqu'il fait référence à d'autres personnes, celles-ci apparaissent dans le discours de ce manager soit par l'évocation de leur profession (journaliste, commerciale), soit par celle de leur fonction (manager, éditeur).

Ne se rendant qu'une fois par an sur le site de son entreprise, son absence a clairement généré un sentiment de distance physique entre lui et ses employés dans l'espace et dans le temps. Le choix des verbes utilisés indique que ses actions ne visaient que les objets non-humains tels que " fixer les objectifs », " détecter les causes », " résoudre le problème » etc. Ce qu'il appelait « management à distance » représente non seulement une distance physique, mais également et surtout une distance émotionnelle dans la relation entre lui et les autres :

J'ai fixé des objectifs stratégiques pour le magazine et aussi pour l'entreprise et ces objectifs stratégiques devraient être (...) pour l'équipe opérationnelle au niveau des journalistes, au niveau de la personne responsable de l'équipe commerciale. Et donc le management se faisait à $90 \%$ à distance...

Nous avons constaté que faute d'avoir placé ses employés au centre de son activité, le manager n'avait pris en compte que l'aspect technique et négligeait l'aspect humain de ses affaires. 


\section{Discours sur l'expérience significative}

Sous forme du 'moment de vérité', l'expérience significative de ce manager intervient lorsque le sujet reconnaît son manque de suivi dans sa tâche managériale. En dépit de toutes les formules de calcul élaborées par cet « ancien ingénieur », il se heurte à des résultats inattendus, contraires à toutes ses attentes. Il s'est agi également d'un début du processus d'apprentissage visant à comprendre l'origine du problème.

Donc j'ai procédé à des entretiens avec les dix centres (...) pas atteints.

En mettant au centre de ses préoccupations, celles des autres sujets, il parvient à réaliser que c'était l'équipe commerciale dont la responsable commerciale, les deux commerciaux ainsi que les clients.

Un deuxième point qui m'a fait changer mon attitude, c'était au niveau de l'équipe commerciale. Au niveau de l'équipe commerciale, il y avait la responsable commerciale qui pilotait deux personnes.

Pour ce manager, l'expérience significative a été le moment où il s'est détourné de l'aspect 'chiffres' au profit de l'aspect humain. A partir de cette réplique, il ne se concentrait plus uniquement sur les personnels en interne, mais aussi sur les personnes externes (clients). De plus, il a su percevoir et comprendre à ce niveau la conséquence de l'absence de lien entre deux individus en raison du manque de communication entre eux.

\section{Discours sur la manière nouvelle/reconstruite}

Dans les discours du cinquième manager, après avoir constaté que les causes étaient dues à la distance entre lui et ses employés (manque d'anticipation, manque de suivi régulier...), les verbes utilisés révèlent une augmentation remarquable de sa présence ou plutôt de sa disponibilité :

Donc ce que j'ai décidé était d'essayer de répartir mon temps de travail entre cette affaire et mes activités ici et aussi de restaurer des réunions régulières, c'est-à-dire des réunions hebdomadaires disons chaque vendredis avec une équipe.

L'expérience significative ne mène pas le sujet directement à la reconstruction de sa manière, mais à la recherche des causes. Ce n'est qu'ensuite, l'analyse des causes le conduit à la reconstruction de sa manière afin d'empêcher que les problèmes ne se reproduisent de nouveau.

Donc ces raisons m'ont permis de changer d'attitude. C'est l'attitude plutôt d'être présent dans l'anticipation et le suivi quotidien et même hebdomadaire. (...) De 
toute façon je leur disais que maintenant je suis disponible si éventuellement il y avait un problème urgent à régler, même ce n'est pas un vendredi et s'il y a une décision stratégique disons à prendre. Voilà.

L'évolution dans le vocabulaire utilisé indique le changement délibéré de son attitude : les longues absences ont été remplacées par une présence plus fréquente, mais planifiée, puis par une disponibilité flexible en fonction des besoins et de la situation. Le point le plus important est la nouvelle capacité de notre sujet à rassurer les autres. Cette capacité lui a permis de stimuler les autres et de changer leur attitude à leur tour.

\section{Discours sur la leçon tirée}

Dans le cinquième cas, le manager a fait une recherche sur les causes pour tirer sa leçon. Etant ingénieur, il a démontré son habitude à s'intéresser aux causes afin de décider des actions éventuelles à entreprendre.

Il faut noter qu'il y avait un certain nombre de causes. Donc déjà, parce qu'à mon niveau, j'avais aussi d'autres activités, qui me prenaient beaucoup de temps donc le suivi à mon niveau n'était pas assez régulier.

Après son autocritique sur son propre engagement, le manager interviewé s'est tourné vers son adjointe, la responsable commerciale. Derrière le fait qu'une personne «était désignée » pour le seconder, notre manager a ainsi pu regarder, dans le détail, le travail de sa collaboratrice et donc voir que « cette personne (...) avait une charge de travail qui était devenue énorme ». Par la suite, son 'analyse' se porte sur les clients, un autre maillon de la 'chaine d'approvisionnement' de son entreprise. Une définition fine de différentes catégories de clients faite par le manager nous a montré la progression de sa compréhension de la nature des activités de chaque sujet humain.

Il faut noter que le magazine, les clients ne sont pas seulement ceux qui achètent le magazine. Et puis le magazine n'est pas mesuré par que des clients, qui achètent le magazine. C'est les (...) publicitaires. Voilà, ces personnes, devaient aller rencontrer les entreprises ou mettre en place les sessions publicitaires. Donc souvent on a vu l'annulation des rendez-vous avec les entreprises, le paiement du (...) publicitaire a tardé.

En retraçant les causes du retard de la production du magazine, le manager a suivi toutes les étapes des activités de ses collaborateurs. A chaque paragraphe, il fait le lien entre une catégorie de personnes et une autre au travers des activités les liant ensemble. Ici les clients sont liés aux journalistes par la prise des rendezvous et des rencontres. L'absence des rendez-vous lie à la production de magazine et aussi au paiement des imprimeurs etc. 


\section{Reconstruire sa manière de faire agir autrui : un apprentissage}

Sur la base des discours tenus par les managers sur leur manière de faire agir autrui dont nous venons de faire état, nous nous attacherons plus particulièrement aux reconstructions que nous pouvons en induire sur la transformation des managers. Notre approche dans cette partie consiste à exposer et à expliquer aux lecteurs les cinq cas dans leur ensemble, en gardant les mêmes quatre thèmes exposés précédemment.

\section{La manière ancienne}

Pour ces cinq cas, au-delà de l'évolution progressive de l'attitude liée à l'ancienneté de chaque individu, ce qui a attiré notre attention est la 'soudaineté' de la rupture du sujet avec l'attitude ancienne/initiale, qui s'est produite à un moment précis ou pendant une courte période.

Leur ancienne manière de faire agir autrui était conditionnée par leurs premières perceptions professionnelles encore immatures, naïves, inexpérimentées et subjectives, y compris la perception qu'ils avaient d'eux-mêmes. A cette logique correspond une évolution incrémentale : les lacunes d'expérience, de compréhension et de confiance en soi se comblent au fil du temps, les perceptions immatures des managers-débutants s'ajustent, la manière ancienne ou initiale de faire agir autrui des managers évolue également, mais sans changer de nature.

Cette manière ancienne/initiale a ainsi été façonnée au travers d'une sorte d'encadrement tel que l'éducation ou la formation reçue, ou encore la hiérarchie, les procédures et la culture propre à l'entreprise. Présentée dans les discours des managers interrogés comme une manière reproduite à partir des 'meilleures pratiques' existantes, la manière ancienne ou initiale est très homogène et procédurale. Elle peut être appliquée dans tout type de contexte et suit un schéma d'étapes prédéfinies. Elle s'appuie sur des outils sous forme écrite, et non pas sous forme orale. Nous n'avons trouvé qu'un minimum, voire une absence d'interaction orale ou expression d'émotion dans leur action.

En appliquant cette manière ancienne, les sujets pouvaient faire agir les autres, mais sans se soucier de la réaction des autres ni de l'impact que leur façon de faire peut avoir sur les émotions des autres. L'encadrement par des modèles prédéfinis les avait poussés vers un mode de fonctionnement par défaut, normalisé, sans personnalité ni singularité, prêt à être utilisée par quiconque occupant ce poste dans l'organisation.

\section{L’expérience significative}

Nous avons été étonnée par la capacité de s'identifier à autrui qui transparait dans ce que ces managers vivent et ressentent. 
Les expériences évoquées comportent une forte dimension émotionnelle : ils sont tous marqués par des sentiments négatifs ressentis par le sujet de la part d'une autre personne : peur, colère, angoisse, inquiétude, déception.

L'expérience significative s'est agie d'une prise de décision délibérée et rapide. Toutes les situations ont justifié et encouragé les sujets à rompre avec leurs habitudes d'action. Les sujets ont été forcés de « devenir autre » dans leur manière de travailler et de faire agir les autres.

Elle constitue en même temps un risque et une opportunité pour un individu de montrer/prouver sa capacité de faire agir autrui. Cet effet d'autonomie professionnelle peut être le résultat d'une absence accidentelle, imprévue de la personne appropriée à un moment critique. Plus le sujet s'éloigne de l'encadrement habituel tel que la formation ou de la hiérarchie de son entreprise, plus il rompt facilement avec son ancienne manière.

Par conséquent, nous avons vu qu'une situation de vie professionnelle, qui aurait pu rester banale, ordinaire et oubliée comme beaucoup d'autres situations similaires, est devenu une expérience significative stimulant une attitude d'apprentissage de la part d'un sujet prêt à faire face à tous types de résultats issus de sa décision.

\section{La manière d'agir nouvelle}

Si la manière d'agir ancienne paraissait rationnelle et généralisable, la manière nouvelle présente un caractère spontané et intuitif. Spontanée, car la manière nouvelle pouvait être adoptée par le manager rapidement, sans longue réflexion. Intuitive, parce qu'au moment où nos managers ont choisi d'agir contrairement à la manière habituelle/initiale, la raison de leur choix n'était pas évidente, ni même explicable. Pour chacun, la nouvelle façon d'agir était complètement inédite, c'està-dire ni prédéfinie, ni transférée par d'autres.

Un constat important est que dans les cinq cas, les discours de nos managers ont montré que la manière nouvelle/reconstruite comportait systématiquement une dimension de communication orale, d'interaction entre le sujet et autrui. En même temps, cette dimension était très informelle et ne pouvait être décrite par des étapes ou remplacée par un courriel, un manuel ou un quelconque autre mécanisme automatisé ou programmé.

Par ailleurs, cette manière comporte un caractère personnel et unique. Elle diffère d'un manager à l'autre et peut être appliquée uniquement par le sujet en question, à un moment donné et selon la nécessité sortie du contexte. Elle est unique et ne peut être imitée, car elle dépend de nombreux paramètres contextuels et variés, notamment de la réaction des autres et son impact sur l'activité de ces derniers. 


\section{Leçon tirée de l'expérience significative}

En comparant les objets de préoccupation de ces managers, nous avons remarqué un changement ou une reconstruction. Dans un premier temps, les objets de leur travail étaient tangibles, concrets et individuels, mais dans un deuxième temps et après l'évènement d'influence, ils étaient devenus plus conceptuels, émotionnels et relationnels.

Le point commun est que ces managers ont commencé à se soucier de la formalisation de valeurs émotionnelles et relationnelles. Nous avons en effet pu détecter un changement dans leurs objectifs, qui a ensuite influencé la reconstruction de leur manière de faire agir autrui. Grâce à ces points distinguant de nos cinq cas des autres cas, nous sommes désormais en mesure de comprendre quelle pourrait être la différence entre un apprentissage graduel par formation formelle et un apprentissage par reconstruction dans l'expérience.

\section{Expérience significative et transformation}

\section{Apprentissage par réflexion sur l'expérience}

Si de plus en plus de types d'apprentissage sont reconnus, ce foisonnement nous fait 'soupçonner' l'existence d'une multitude d'autres formes d'apprentissage qui ne sont pas encore 'formalisées'. En passant d'une approche inductive basée sur nos remarques tirées de conversations et d'observations professionnelles à une approche déductive basée sur les théories d'apprentissage social, nous avons pu conclure que parmi les nombreux changements survenus dans le monde de travail en entreprise, il existe un phénomène qui amène certains managers à reconstruire leur manière d'agir sur l'activité d'autrui dans leurs interactions avec les autres sujets humains.

En général, les manières de faire agir autrui adoptées par les managers peuvent être classées en plusieurs catégories : le commandement, la négociation, la motivation, la manipulation... etc. Si le choix habituel d'une manière dépend aussi de données personnelles ou de l'éducation, le passage de cette manière 'primaire' habituelle à une manière 'secondaire' peut être soit le résultat d'une évolution lente et progressive soit d'un processus délibéré d'autoréflexion et de prise de décision. Ce processus qui pousse un manager à abandonner, à remplacer sa manière d'agir habituelle et bien définie par une autre manière nouvelle et reconstruite est la forme d'apprentissage par réflexion sur l'expérience significative du manager. 


\section{L'émotion dans l'apprentissage}

D'une part, après certaines recherches en psychologie 5 , l'émotion peut 'naître' à la suite d'une expérience significative. Quant à nous et sous l'angle de l'analyse des activités $^{6}$, nous pensons que bien qu'il n'y ait pas d'action sans émotion, seules certaines émotions sont productives de transformations. En outre, parmi de nombreuses expériences de la vie quotidienne, si une expérience ordinaire peut ou ne peut pas générer des émotions, la réflexion sur une expérience ordinaire peut y contribuer. Sur cette base, le couple émotion/réflexion doit pouvoir rendre une expérience banale de la vie quotidienne plus informative, cognitive, psychique et significative pour le manager. En d'autres termes, l'émotion dans l'apprentissage des attitudes chez les managers n'est pas seulement une forme de rétroaction, mais aussi un système d'interprétation en perpétuel mouvement.

D'autre part, comme une forme de l'émotion, l'affect peut générer de la 'frustration' et stimuler le changement d'attitude du sujet. Le sentiment d'infériorité d'un sujet, face à son 'concurrent', peut le conduire à 'critiquer' ou 'rejeter' tout ce qui est lié à la source perçue de cette 'frustration'. Cependant, ce même affect, dans une certaine mesure, peut se transformer en un 'stimulateur' qui conduit le sujet à 'agir différemment', par exemple s'ouvrir aux autres, accepter les critiques et produire de nouvelles pratiques ou habitudes d'activité. Les sujets peuvent agir pour changer d'attitude afin d'atténuer ou d'aggraver la dissonance formée en raison du conflit entre attitude et comportement. La dissonance pousse le sujet à agir pour rétablir la consonance et sortir de la dissonance même s'il doit reconstruire sa manière de travailler habituelle.

\section{Conclusion générale}

En définitive, les facteurs clés ne sont pas les mêmes pour l'acquisition de compétences techniques que pour celle de compétences humaines. La dissonance et l'émotion, qui jouent un rôle secondaire dans l'apprentissage de ces premières, jouent un rôle décisif dans la reconstruction des manières de faire agir autrui des managers. Cette conclusion nous permet de contribuer à une meilleure appréhension à la fois du travail des managers et de l'apprentissage expérientiel des adultes.

\footnotetext{
5 « Les émotions émergent des processus biologiques, mais également du traitement de l'information, des interactions sociales et des contextes culturels » (Reeve 2015 : 340). Selon Jonhmarschall Reeve, l'émotion comprend quatre composantes qui se manifestent sous différentes formes et aident l'individu à agir : "Les émotions sont des réactions sentimentales, éphémères, intentionnelles, expressives, corporelles, qui nous aident à nous adapter aux opportunités et aux défis auxquels nous sommes confrontés lors d'événements importants dans la vie » (ibidem : 452).

${ }^{6}$ Jean-Marie Barbier définit (il y a plusieurs auteurs) les émotions comme un « Eprouvé psychique lié à la singularité d'une situation pour un sujet, opérant une rupture dans le cours habituel de son activité » (Barbier et all. 2011 : 57).
} 


\section{Bibliographie}

Barbier J.-M., Chauvigné, C., Vitali, M.-L. (2011) Diriger : un travail. Paris : L'Harmattan.

Drucker P. (2012) Management, London and New York : Routledge, Taylor \& Francis Group.

Festinger L. (1962) A Theory of Cognitive Dissonance, Stanford, California, Stanford University Press, https://doi.org/10.1038/scientificamerican1062-93

Reeve J. (2015) Understanding motivation and emotion. Hoboken, NJ, Wiley.

Saget M. L. (2013) Le Manager Intuitif, Paris, Dunod.

Seignour A. (2011) Méthode d'analyse des discours. L'exemple de l'allocution d'un dirigeant d'entreprise publique, "Revue Française de Gestion », 2 (211) : 29-45, https://doi.org/10.3166/rfg.211.29-45

Vu K. (2020) Reconstruire les manières de faire agir autrui : un apprentissage des managers à partir de leur expérience, thèse de Doctorat en sciences de l'éducation, Paris, Cnam, France.

\section{Changing One's Ways of Making Others Act after Significant Experiences: the Case of Managers}

\section{Summary}

Faced with the question that many researchers in the fields of management and education are asking: how are the human competences of managers made, it seems essential to study the process by which adults learn their leadership by changing the way they make others act, and thus acquire human competences to carry out their activity as managers. The objective of this article is to present to the readers particular situations in which we can observe important changes in the way adult subjects make others act.

With empirical work on adults' testimonies of their interactions with others, this article describes the processes and activities that characterize this self-change of managers in their relationships with others. Referring to the grounded theory and the Chicago School approach, an inductive and qualitative method was adopted, consisting of in-depth research with managers who had lived through such significant experiences. The research was based on the identification of four types of information: the former way of acting, the reconstructed way of acting, the significant experience, and lessons learned from the significant experience. 
The results of the research, presented in the article, allow for a better understanding of the difference between progressive learning through professionalization and learning through the reconstruction of managers' ways of acting. They also allowed us to observe that the occurrence of these changes depends on two types of factors: the emotions generated by the interactions affecting the managers' very existence, and the managers' commitment to reflect on this experience.

In conclusion, it leads to new perspectives for action and research in terms of training, professionalization and the exercise of the managerial profession.

\section{Zmiana sposobów postępowania wobec drugiego związana ze znaczącymi doświadczeniami: przypadek menadżerów}

\section{Streszczenie}

Wielu badaczy z obszarów zarządzania i edukacji stawia pytanie o to, jak kształtować kompetencje menadżerów. Wydaje się ważne przestudiowanie tego procesu, w którym dorośli uczą się przywództwa, kierowania innymi, zmieniając sposoby oddziaływania na drugiego i tym samym nabywając humanistycznych kompetencji w realizowaniu aktywności menadżera. Celem tego artykułu jest przedstawienie czytelnikom wybranych sytuacji, w których można zaobserwować istotne zmiany w sposobach oddziaływania na innych.

Na podstawie materiałów empirycznych zebranych wśród dorosłych, na temat ich interakcji z innymi, opisano procesy i aktywności charakteryzujące zmiany menadżerów jako siebie samych, $\mathrm{w}$ relacjach $\mathrm{z}$ innymi. Odnosząc się do teorii ugruntowanej i podejścia Szkoły Chicagowskiej, zastosowano metodę indukcyjną i jakościową, stanowiącą pogłębione badania $\mathrm{z}$ kadrą kierowniczą posiadającą przeżycia takich znaczących doświadczeń. Badanie opierało się na identyfikacji czterech typów informacji: dawny sposób postępowania, zrekonstruowany sposób postępowania, doświadczenie znaczące, nauka płynąca z tego doświadczenia.

Rezultaty badania przedstawione $\mathrm{w}$ tym artykule pozwalają lepiej zrozumieć różnicę między stopniowym uczeniem się $\mathrm{w}$ toku profesjonalizacji/przygotowania do zawodu a uczeniem się poprzez rekonstrukcję sposobów oddziaływania menadżerów na drugiego. Pozwoliły także zaobserwować, że okoliczności/kontekst tych zmian zależy od dwu typów czynników. Są nimi: emocje generowane poprzez interakcje, które wpływają wręcz na byt/istnienie menadżerów oraz zaangażowanie menadżerów w refleksję nad danym doświadczeniem.

W konkluzji artykułu wskazuje się na nowe perspektywy działania i badań w obszarze kształcenia, profesjonalizacji i wykonywania zawodu menadżera.

\section{Pour citer cet article}

Vu K. (2021) Changer ses manières de faire agir autrui à l'occasion d'expériences significatives : le cas de managers, „Nauki o Wychowaniu. Studia Interdyscyplinarne” 2(13), 205-226, https://doi.org/10.18778/2450-4491.13.13 